\title{
Two-dimensional metallic nanobowl array transferred onto thermoplastic substrates by microwave heating of carbon nanotubes
}

\author{
Tsung-Han Chen ${ }^{1}$, Tsung-Yen Tsai ${ }^{1}$, Kun-Che Hsieh ${ }^{2}$, \\ Shih-Chin Chang ${ }^{1}$, Nyan-Hwa Tai ${ }^{1}$ and Hsuen-Li Chen ${ }^{2}$ \\ ${ }^{1}$ Department of Materials Science and Engineering, National Tsing Hua University, 101, \\ Section 2, Kuang-Fu Road, Hsinchu, Taiwan 30013, Republic of China \\ 2 Department of Materials Science and Engineering, National Taiwan University, 1, \\ Section 4, Roosevelt Road, Taipei, Taiwan 10617, Republic of China \\ E-mail: scchang@mx.nthu.edu.tw
}

Received 7 August 2008, in final form 1 September 2008

Published 21 October 2008

Online at stacks.iop.org/Nano/19/465303

\begin{abstract}
The unique heating property of microwave irradiation on carbon nanotubes (CNTs) was applied in fabrication of a new architecture of two-dimensional metallic nanobowl array on a thermoplastic substrate. Vertically aligned CNT film was grown on a metal coated polystyrene (PS) submicron sphere array and then transferred onto a polycarbonate (PC) substrate by microwave heating. The back diffracted light spectra of this structure were measured and discrepancies between the spectra and that predicted from a diffraction relationship were studied. It is suggested that the discrepancies were related to the shape and the refractive indices of the materials.
\end{abstract}

\section{Introduction}

Recently, periodical nanostructures have attracted a wide interest in both physical and biological fields [1-6]. To make an artificially periodical nanostructure, a typical and effective way is by using self-assembled submicron PS spheres array as the template. Based on it, two-dimensional periodical nanostructures such as nanobowl [7-10], CNT [11, 12], nanorod [13], nanohoneycomb [14], and nanoscale ring [15] arrays had been demonstrated. Among them, the nanobowl array attracted much attention because it can be used as a sieve for selecting nanoparticles, and has the potential for biomedical applications such as nanocontainers. However, up to now, to get the nanobowl structures mentioned above has been either very expensive using atomic layer deposition (ALD) followed by ion-milling [7,8], or quite complicated using chemical process $[9,10]$. Furthermore, the nanobowl structures thus made were mostly built on rigid/stiff substrates so that many useful applications could not be realized.

As reported before, CNTs can be transferred onto thermoplastic polymers by microwave heating [16-18]. The bonding between the CNTs and the polymer substrate was so strong that the CNTs hold the polymer together even when the polymer substrates were broken by the pulling force. In this work, an array of metallic nanobowls was attached to a thermoplastic polymer substrate by applying microwave heating of the CNTs. One of the unique advantages of the flexible substrate was demonstrated by the change of the diffracted colour of a bent specimen and the back diffracted light spectra of the structure were studied.

\section{Experimental details}

The process is as follows. First, a $10 \times 10 \mathrm{~mm}^{2}$ p-type $\operatorname{Si}(100)$ wafer was cleaned with deionized water in ultrasonic bath for $10 \mathrm{~min}$ and then hydrophilic treated at $70^{\circ} \mathrm{C}$ for $45 \mathrm{~min}$. The solution for hydrophilic treatment was made of $500 \mathrm{ml}$ deionized water, $100 \mathrm{ml}$ of hydrogen peroxide $\left(\mathrm{H}_{2} \mathrm{O}_{2}, 26 \%\right)$ and $100 \mathrm{ml}$ of ammonia $\left(\mathrm{NH}_{4} \mathrm{OH}, 38 \%\right)$. A $10 \mathrm{wt} \%$ solution of monodisperse PS spheres (Microparticles GmbH, Germany) was mixed with methanol at a volume ratio of $4: 1$. A drop of the mixed solution 15-20 $\mu 1$ was applied on the hydrophilic treated Si wafer surface. Then by spinning at a controlled speed of $800 \mathrm{rpm}$ for $60 \mathrm{~s}$, a monolayer of close-packed PS sphere 
(I)

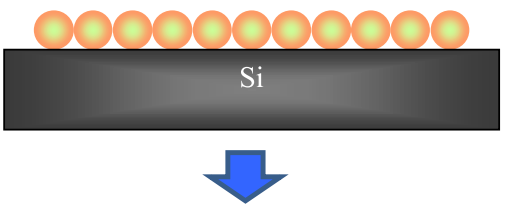

(II)

00000000000

$\mathrm{Si}$

(III)
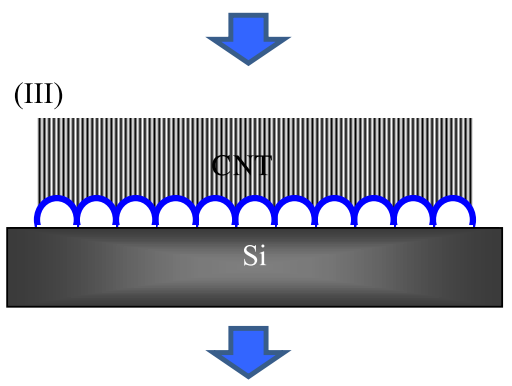

(IV)

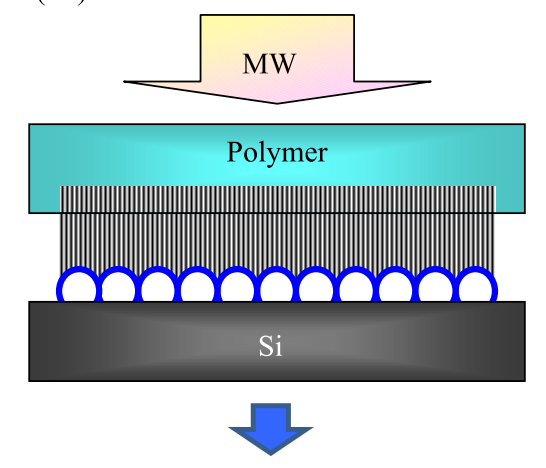

(V)

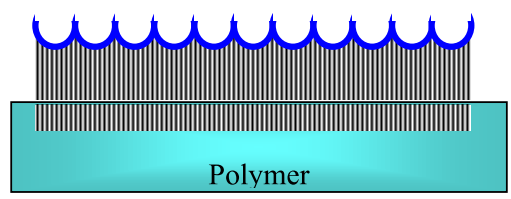

Figure 1. The fabrication of a two-dimensional metallic nanobowl array on a thermoplastic substrate: (I) A monolayer of PS sphere array is formed on a Si wafer by the spin-coating method. (II) Co/Ti/TiN/Mo coatings were deposited on PS sphere by sputtering. (III) The PS spheres were evaporated out and vertically aligned CNT film was grown on the metallic coating. (IV) A piece of thermoplastic PC was put on and welded to the CNT film by microwave heating. (V) The Si wafer was removed simply by a pair of tweezers and an artificial nanostructure of metallic nanobowl array on a thermoplastic substrate was obtained.

(This figure is in colour only in the electronic version)
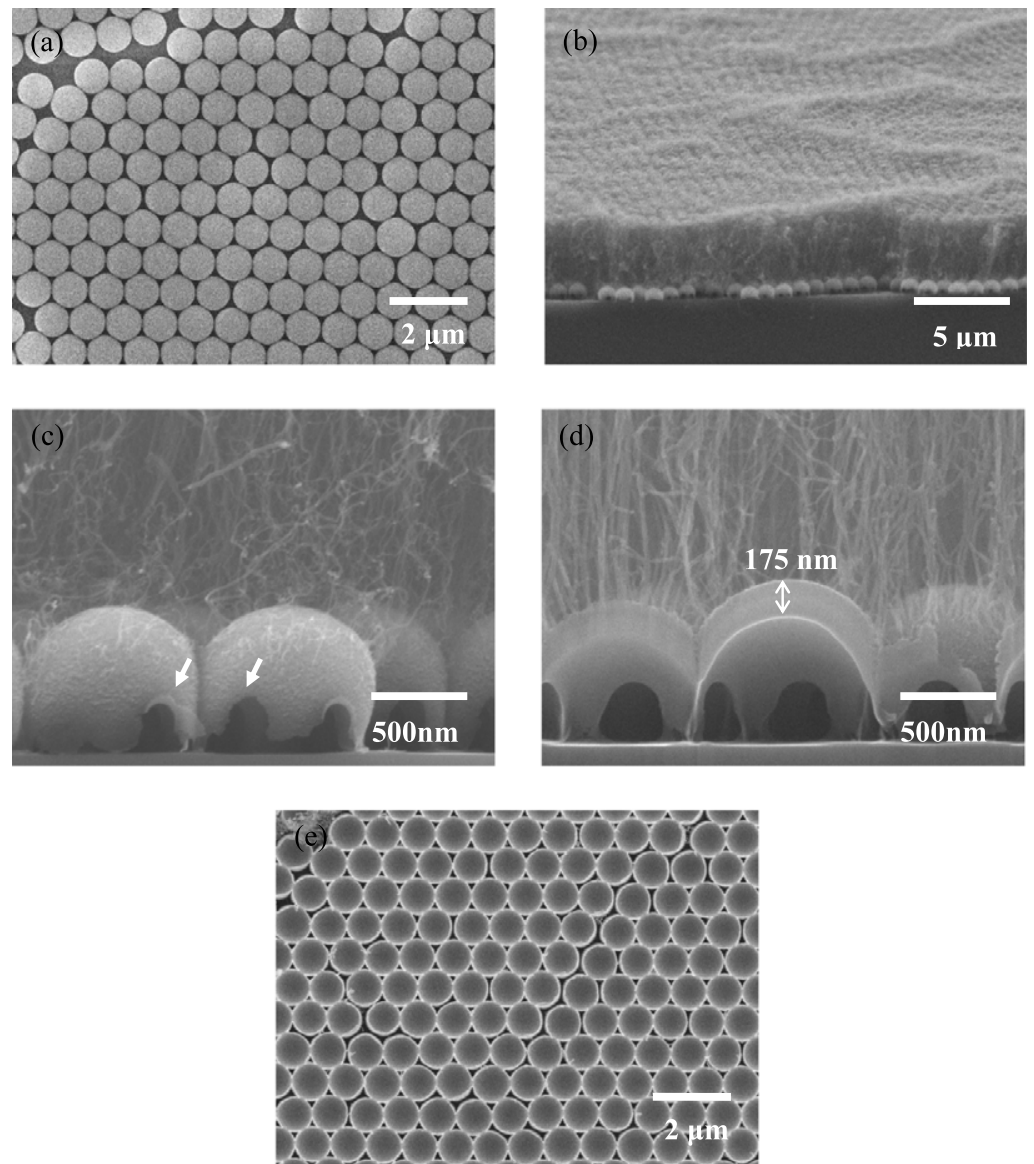

Figure 2. (a) Scanning electron microscopy (SEM) image of a close-packed monolayer of PS spheres settled onto a silicon wafer. (b)-(d) The cross-section of the specimen after carbon nanotube growth process: (b) a $30^{\circ}$ side view, (c) higher magnification, (d) a cross-section cut with focus ion beam (FIB), and (e) a top view of the metallic nanobowls transferred onto a PC substrate. 

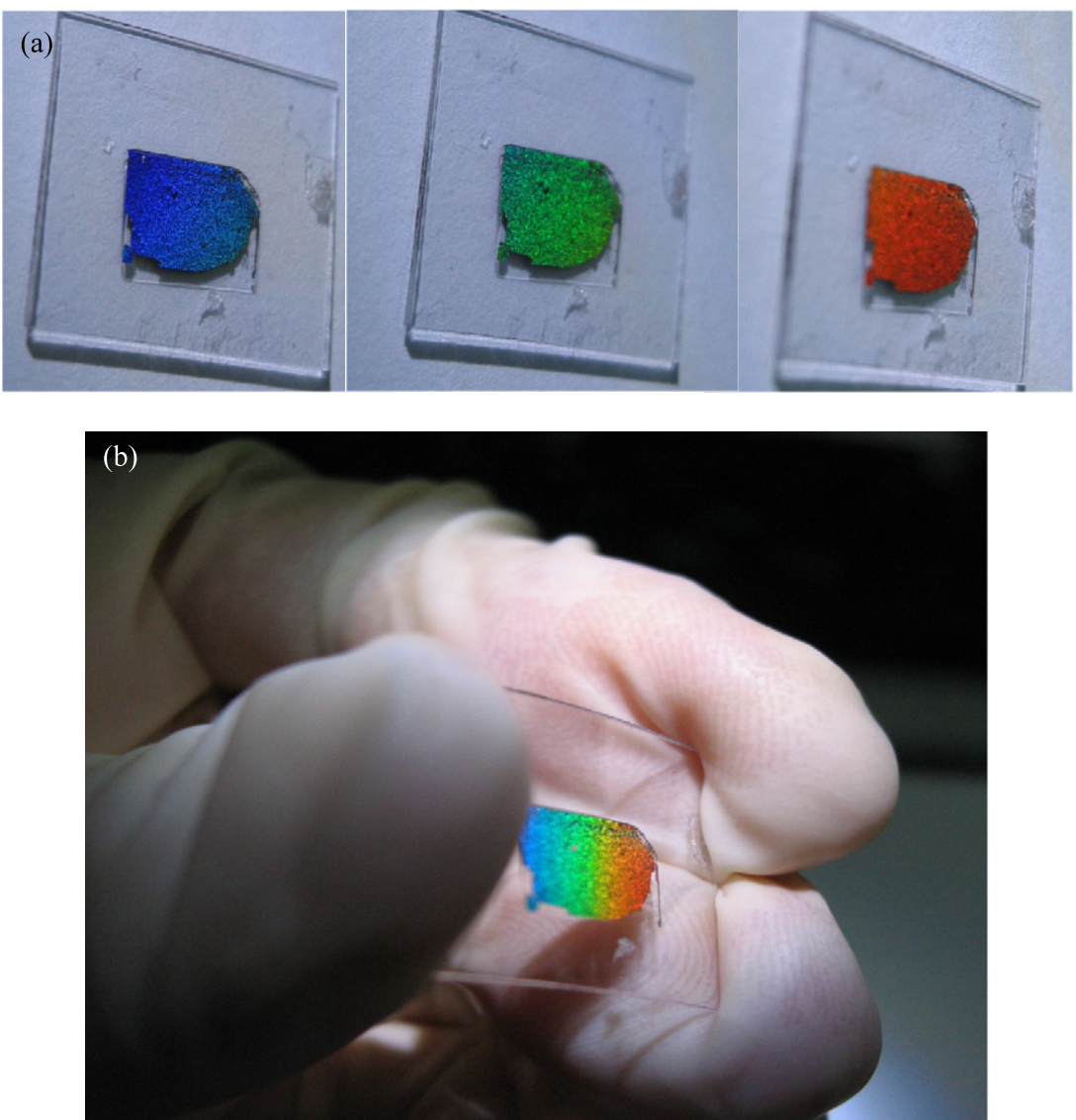

Figure 3. (a) The iridescent colour of the specimen changes with the view angle. (b) A continuous spectrum of colours was observed on the specimen bent between fingers.

array can be obtained on the Si wafer. A multilayer coating of $5 \mathrm{~nm} \mathrm{Co/1} \mathrm{nm} \mathrm{Ti/75} \mathrm{nm} \mathrm{TiN/100} \mathrm{nm} \mathrm{Mo} \mathrm{was} \mathrm{deposited}$ on the PS spheres array by sputtering. Then the CNTs were grown by rapid heating chemical vapour deposition (CVD) process as reported elsewhere [19]. In the process, the PS spheres array with multilayer coating was heated to and kept at $600{ }^{\circ} \mathrm{C}$ for 5 min under a low pressure of 10 Torr with a flow of $30 \mathrm{sccm} 10 \% \mathrm{H}_{2}$ /Ar mixture before the carbon source (acetylene) was introduced into the CVD chamber. In this way, the PS $\left(T_{\text {melt }}=240^{\circ} \mathrm{C}\right)$ spheres were melted and evaporated out completely before the growth of the CNTs and no residue of the PS was found in the specimen.

To transfer the CNT film, a thermoplastic polymer substrate such as a PC film (500 $\mu \mathrm{m}$ in thickness) was placed on top of the vertically aligned CNT film and put in a 2.45 $\mathrm{GHz}$ microwave reaction chamber. The reaction chamber was mechanically evacuated to $1 \times 10^{-3}$ Torr and then refilled with nitrogen gas of 500 Torr. The microwave generator was operated at $400 \mathrm{~W}$ for $30-60 \mathrm{~s}$ to have the CNTs fixed to the PC film. After the microwave heating, the Si wafer could be removed simply by a pair of tweezers and an artificial nanostructure of metallic nanobowl array on a thermoplastic substrate was obtained. Figure 1 is a step by step illustration of the whole process.

The specimens were then examined with a field-emission scanning electron microscope (FE-SEM, JEOL-6500F). The optical properties of periodic nanostructures were measured by a UV-vis spectrophotometer (Hitachi, U-4100) with varied detector angles under normal incident light. Focus ion beam (FIB, FEI Quanta 200 DBFIB) operated at $30 \mathrm{kV}, 100 \mathrm{pA}$ was used to cut the sample at an incident angle of $90^{\circ}$ to the sample surface.

\section{Results and discussion}

Microwave heating of materials is unique in that the heating is very fast, selective and localized. When CNTs and thermoplastic polymers such as PC, PET, etc are microwave irradiated together (figure 1, step IV), the temperature of the CNTs will increase drastically in just a few seconds while the thermoplastic polymers are almost unaffected. The hot CNTs pierced the thermoplastic polymers just like hot steel wires piercing a super-cooled chunk of ice. As soon as the heating power of the wires was turned off, the super-cooled chunk of ice would refreeze the small local melt part immediately and would hold the steel wire firmly. And so was the thermoplastic substrate held the CNTs firmly.

Figure 2 is the scanning electron microscopy (SEM) images of the specimen. Figure 2(a) shows the monolayer film of PS spheres formed on a $10 \times 10 \mathrm{~mm}^{2}$ silicon wafer. The film contains domains of perfect close-packed spheres and defects such as vacancies and cracks. The domain size 
ranges from 10 to $30 \mu \mathrm{m}$. The diameter of the PS spheres was measured to be $905 \pm 5 \mathrm{~nm}$ before the deposition of the metallic coating. Figure 2(b) is a $30^{\circ}$ side view SEM image of the specimen after the carbon nanotube growth process. The CNT film grown on the coated spheres is vertically aligned and the thickness of the CNT film is $3.5 \mu \mathrm{m}$. Since the sputtered metal was coated on the upper but not all the lower half of the PS spheres, arched channels between neighbouring spheres were observed (indicated by white arrows in figure 2(c)). When the specimen was heated to $600{ }^{\circ} \mathrm{C}$ under low pressure before the CNT growing process, the PS spheres were easily melted and evaporated out and left the hemispherical metallic shell on the Si substrate as shown in figure 2(c). Figure 2(d) is a SEM image of the specimen cross-section cut by focus ion beam (FIB), in which one can see the thickness of the metallic shell is about $175 \mathrm{~nm}$.

Figure 2(e) shows the top view of the CNT film with metallic hemispherical shell transferred onto the PC substrate by microwave heating. The CNT film with hemispherical metallic shells concave upward arranged as an array of two-dimensional close-packed nanobowls. The diameter of nanobowls on this specimen was measured to be $875 \pm 5 \mathrm{~nm}$, about 3\% smaller than that of the PS sphere measured before CNT growth. Different sized nanobowls can be produced by using different sized PS spheres.

When one looks at the specimen from different angles, the iridescent colour of it changes as shown in figure 3(a). Figure 3(b) shows an optical image of the specimen bent between fingers to emphasize the flexibility of the specimen. A continuous spectrum of colour was observed on it due to the continuous change of the view angle.

Figure 4(a) shows the reflectance spectrum of the $875 \mathrm{~nm}$ nanobowl array measured by a detector at an angle of $60^{\circ}$ with the incident light normal to the specimen. The measured range of the spectrum wavelength was from 300 to $800 \mathrm{~nm}$. There are three peak wavelengths on the spectrum, namely 710, 408 and $355 \mathrm{~nm}$. The ratio of these three peak wavelengths is $1: 1 / \sqrt{ } 3$ : $1 / 2$ which is exactly the ratio of the distances between the first three most dense rows in a two-dimensional close-packed array. Similar spectra were observed for different detector angles and nanobowl sizes. Figure 4(b) shows the reflectance spectra of the $875 \mathrm{~nm}$ nanobowl array measured by a detector at angles of $40^{\circ}, 50^{\circ}, 60^{\circ}, 70^{\circ}$ and $80^{\circ}$. The peaks in each spectrum shifted to longer wavelengths with the increase of the detector angle. Figure 4(c) shows the reflectance spectra of a $466 \mathrm{~nm}$ nanobowl array. The ratios of the corresponding peak wavelengths of the two different nanobowls size specimens at each detected angles equal to their size ratio, i.e. 466/875 = 0.533 . From all these evidences, it is concluded that the colour of the specimen comes from its periodical nanostructure origin.

It is expected that the diffraction equation $(\sqrt{ } 3 / 2) D$ $\sin \theta=m \lambda=\left(m_{1}^{2}-m_{1} m_{2}+m_{2}^{2}\right)^{1 / 2} \lambda$ for a two-dimensional close-packed array can be used to express the observed peak data in this case perfectly [20]. However, as shown in figure 5(a), there are obvious discrepancies in the order of $8.4 \%$ between the measured peak wavelengths and the counter parts calculated by the diffraction equation based on the measured D. This kind of discrepancy has been reported by several
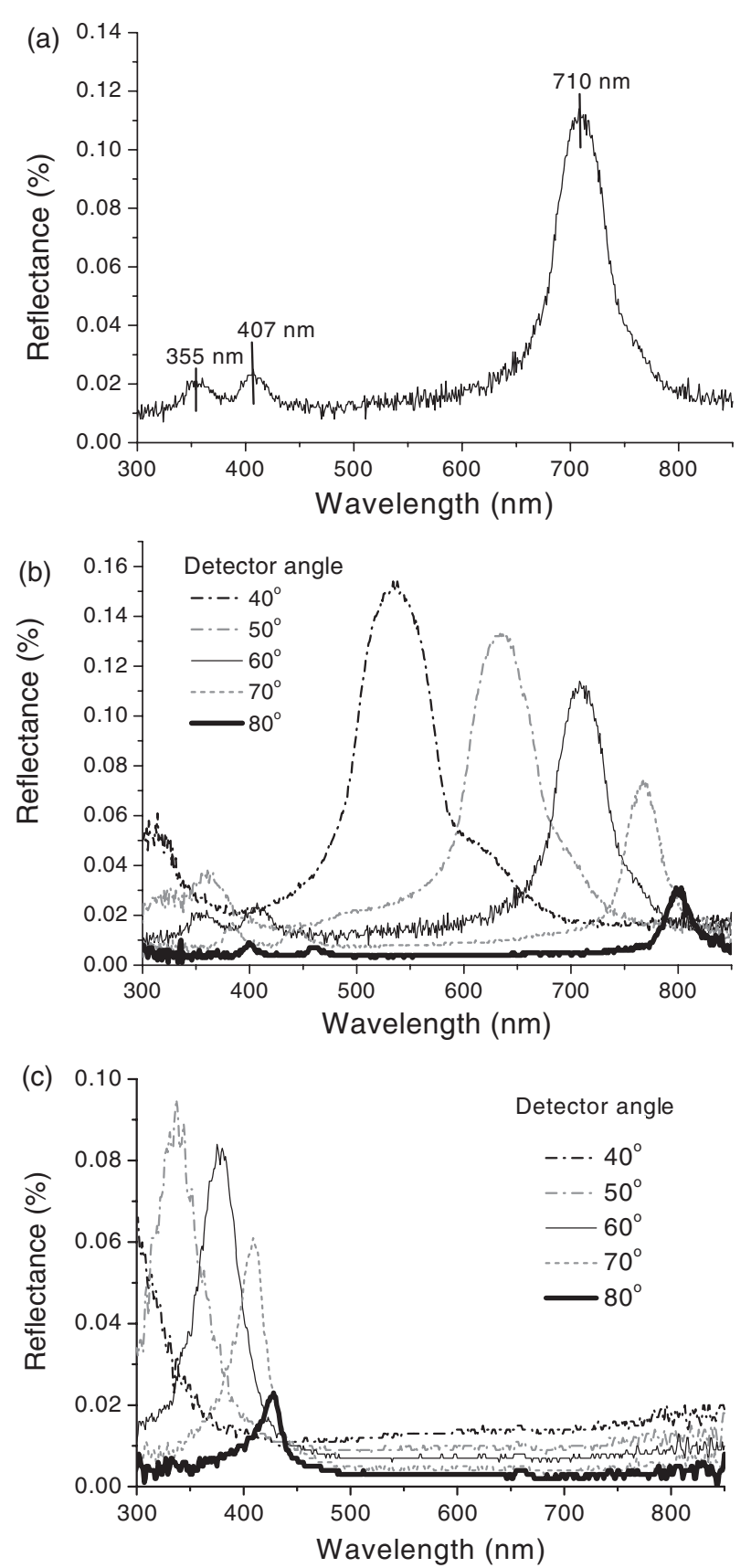

Figure 4. (a) Reflectance spectrum of $875 \mathrm{~nm}$ nanobowl array at $60^{\circ}$ detector angle. (b) and (c) Reflectance spectra of the nanobowl array of different detector angles: (b) $875 \mathrm{~nm}$ nanobowl and (c) $466 \mathrm{~nm}$ nanobowl.

previous workers on two-dimensional close-packed array of PS spheres and they attributed the discrepancies either to experimental errors or to the shrinkage of the PS spheres used in the structures [21, 22]. In order to clarify this problem, many repeated works have been done by the authors to exclude any possible experimental errors large enough to cause the observed discrepancies. We can confidently say that this large discrepancy is not caused by experimental error. After that, three two-dimensional close-packed arrays made of different materials were studied. They are $100 \mathrm{~nm}$ Mo coated hemispherical metallic shell array, $75 \mathrm{~nm}$ TiN coated 

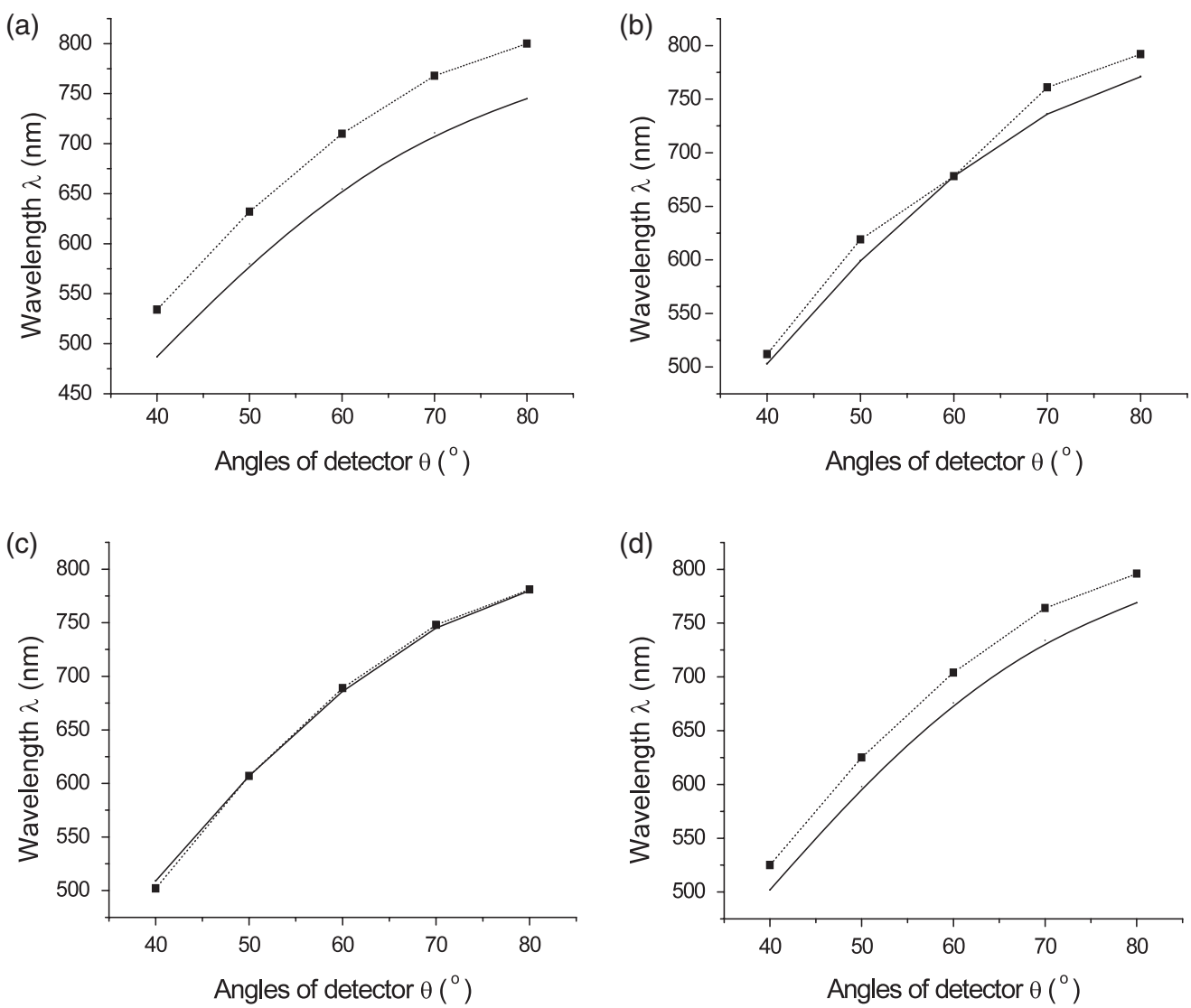

Figure 5. The calculated (solid) and measured (dot) reflectance peak wavelengths $(\lambda)$ of different detector angles $(\theta)$ for: (a) the specimen of transferred nanobowl array, (b) PS sphere array, (c) TiN coated shell array, and (d) Mo coated shell array.

hemispherical metallic shell array, and PS sphere array. It was found that for the PS sphere array, the discrepancy was in the order of $3.4 \%$ (figure 5(b)) which has also been reported by other researchers as mentioned before [21, 22]. In contrast, almost zero discrepancy was found for the TiN coated shell array as shown in figure 5(c) and for the Mo coated shell array, the discrepancy was in the order of $4.2 \%$ as shown in figure 5(d).

Since the diameters of those periodic nanostructures were measured directly by using the standard scale bar of SEM, the discrepancies should not be from the shrinkage of the nanostructures. Despite the difference between the shapes of bowl on CNTs and hemisphere, the major difference among the above arrays is the composition and thus the refractive index of the materials. For light wavelengths from 500 to $800 \mathrm{~nm}$ the refractive index of Mo is from 3.6 to 3.8. In contrast, the refractive index of $\mathrm{TiN}$ is from 1.1 to 0.8 (compare to $\sim 1$ for air) and the refractive index of PS is 1.57 . Therefore, it is suggested that the discrepancies between calculated and measured peak wavelengths may relate to the geometrical shape and the refractive index of the composition materials.

\section{Conclusion}

In conclusion, the unique heating property of microwave irradiation on CNTs was applied in fabrication of a new architecture of two-dimensional metallic nanobowl array on a thermoplastic substrate. In the process, vertically aligned CNT film was grown on metal coated PS submicron sphere array and then transferred onto a polycarbonate substrate by microwave heating. Due to the selective heating characteristic of microwave irradiation, the metallic nanobowl array was fixed firmly on the polymer substrate by the CNTs. Light can be diffracted by this structure and the discrepancies between the calculated and measured diffraction results were suggested to be related to the shape and the refractive index of the composition materials.

\section{Acknowledgment}

The authors are grateful to the National Science Council, Taiwan for the support of this research under NSC 96-2221E-007-101.

\section{References}

[1] Vlasov Y A, Bo X Z, Sturm J C and Norris D J 2001 Nature 414289

[2] Arsenault A C et al 2006 Nat. Mater. 5179

[3] Tan T L, Wong D and Lee R 2004 Opt. Express 124847

[4] Jewell S A, Vukusic P and Poberts N W 2007 New J. Phys. 999

[5] Huang J Y, Wang X D and Wang Z L 2006 Nano Lett. 62325

[6] Fujii S, Ryan A J and Armes S P 2006 J. Am. Chem. Soc. 1287882 
[7] Wang X D, Gruagnard E, King J S, Wang Z L and Summers C J 2004 Nano Lett. 42223

[8] Wang X D, Lao C, Gruagnard E, Summers C J and Wang Z L 2005 Nano Lett. 51784

[9] Li Y, Li C C, Cho S O, Duan G T and Cai W P 2007 Langmuir 239802

[10] Srivastava A K, Madhavi S, White T J and Ramanujan R V 2005 J. Mater. Chem. 154424

[11] Kempa K et al 2003 Nano Lett. 313

[12] Rybczynski J, Kempa K, Wang Y, Zen Z F, Carlson J B, Kimball B R and Benham G 2006 Appl. Phys. Lett. 88203122

[13] Wang X, Neff C, Graugnard E, Ding Y, King J S, Pranger L A, Tannenbaum R, Wang Z L and Summers C J 2005 Adv. Mater. 172103

[14] Kei C C, Chen T H, Chang C M, Su C Y, Lee C T, Hsiao C N, Chang S C and Perng T P 2007 Chem. Mater. 195833
[15] Kosiorek A, Kandulski W, Glaczynsha H and Giersig M 2005 Small 1439

[16] Zhang M, Fang S, Zakhidov A A, Lee S B, Aliev A E, Williams C D, Athinson K R and Baughman R H 2005 Science 3091215

[17] Wang C Y, Chen T H, Chang S C, Cheng S Y and Chin T S 2007 Adv. Funct. Mater. 171979

[18] Wang C Y, Chen T H, Chang S C, Chin T S and Cheng S Y 2007 Appl. Phys. Lett. 90103111

[19] Chiu C C, Tsai T Y and Tai N H 2006 Nanotechnology 172840

[20] Krieger I M and O'Neill F M 1968 J. Am. Chem. Soc. 903114

[21] Duffel B V, Ras R H A, De Schryver F C and Schoonheydt R A 2001 J. Mater. Chem. 113333

[22] Ishii M, Harada M, Tsukigase A and Nakamura H 2007 J. Opt. A: Pure Appl. Opt. $9 \mathrm{~S} 372$ 